\title{
Near-IR photometry of asymptotic giant branch stars in the dwarf elliptical galaxy NGC $147^{\star, \star \star}$
}

\author{
Y.-J. Sohn ${ }^{1,2}$, A. Kang ${ }^{1,2}$, J. Rhee ${ }^{1,2}$, M. Shin ${ }^{1}$, M.-S. Chun ${ }^{1}$, and Ho-Il Kim ${ }^{3}$ \\ 1 Department of Astronomy, Yonsei University, Seoul 120-749, Korea \\ e-mail: sohnyj@yonsei.ac.kr; breeze82@galaxy.yonsei.ac.kr \\ 2 Center for Space Astrophysics, Yonsei University, Seoul 120-749, Korea \\ 3 Korea Astronomy and Space Science Institute, Daejeon 305-348, Korea
}

Received 7 June 2005 / Accepted 31 July 2005

\section{ABSTRACT}

Near-infrared $J, H$ and $K^{\prime}$ images were used to investigate the stellar contents of the asymptotic giant branch (AGB) population in the nearby dwarf elliptical galaxy NGC 147. The obtained $(K, J-K)$ and $(K, H-K)$ color-magnitude diagrams contain stars of AGB and red giant branch populations, where the former consists of a group of bright blue stars, a dominant population of $\mathrm{M}$ giant and a red $\mathrm{C}$ star population. We identified 91 AGB C stars in NGC 147 with the mean absolute magnitude and colors of $\left\langle M_{K}\right\rangle=-7.56,\left\langle(J-K)_{0}\right\rangle=1.81$ and $\left\langle(H-K)_{0}\right\rangle=0.74$. The estimated number ratio of $\mathrm{C}$ stars to $\mathrm{M}$ giant stars $(\mathrm{C} / \mathrm{M})$ is $0.16 \pm 0.02$. The estimated local $\mathrm{C} / \mathrm{M}$ ratios of $0.14 \pm 0.02$ for the inner region $\left(r<70^{\prime \prime}\right)$ and $0.19 \pm 0.03$ for the outer region $\left(r>70^{\prime \prime}\right)$ indicate a weak radial gradient. The mean bolometric magnitude of $91 \mathrm{C}$ stars in NGC 147 is $\left\langle M_{\text {bol }}\right\rangle=-4.32 \pm 0.49$. The bolometric luminosity function of M giant stars in NGC 147 extends up to $M_{\text {bol }}=-5.8$ mag, and that of only $\mathrm{C}$ stars spans $-5.6<M_{\mathrm{bol}}<-3.5$. The color histograms of AGB stars in $(J-K)$ and $(H-K)$ show a main peak containing M giant stars, a red tail containing $\mathrm{C}$ stars with a weak excess, and a blue excess possibly due to bright blue foreground stars and AGB stars younger than $\mathrm{M}$ giants. The comparison of the theoretical isochrone models with the color distribution and the brightness of AGB stars indicates that most of the bright M giants in NGC 147 were formed at $\log \left(t_{\mathrm{yr}}\right) \sim 8.4$ with a range of $8.2 \sim 8.6$. Ages of the majority of M giant stars in NGC 147 are similar to those of younger M giant stars in its companion NGC 185, while their formation occurred more recently than the older M giant stars in NGC 185 by $\sim 2$ Gyr.

Key words. galaxies: individual: NGC 147 - galaxies: stellar content - stars: AGB and post-AGB

\section{Introduction}

Photometric studies of stars resolved in dwarf galaxies, which are among the most common galactic systems in the universe, provide clues for stellar evolution and star formation history in the galaxies and hence the evolution of the systems. However, detailed stellar population studies in dwarf galaxies are possible only for the nearby galaxies in Local Group, due to low luminosity and small size of the systems. Among the resolved bright giants in nearby dwarf galaxies, asymptotic giant branch (AGB) stars make it possible to study the late evolutionary stage of stars with low to intermediate masses $\left(\sim 0.8-8 M_{\odot}\right)$, and the star formation history in a galaxy for the intermediate age of $1 \sim 10$ Gyr (Grebel 1999).

^ Based on observations carried out at the Canada-France-Hawaii Telescope, operated by the National Research Council of Canada, the Centre National de la Recherche Scientifique de France, and the University of Hawaii.

$\star \star$ Full Table 2 is only available in electronic form at the CDS via anonymous ftp to cdsarc.u-strasbg.fr $(130.79 .128 .5)$ or via http://cdsweb.u-strasbg.fr/cgi-bin/qcat?J/A+A/445/69
During the ascent of the AGB that have already passed through core helium-burning phase, stars experience a longperiod pulsation variability, a heavy mass loss, and a change of atmospheric chemical composition. On the evolutionary phase of the thermal pulses (TP), the AGB star is able to undergo a "third dredge-up" process (Iben \& Renzini 1983), which brings ${ }^{12} \mathrm{C}$ and $s$-process elements to the stellar surface (Brewer et al. 1996). This evolutionary mechanism changes the atmospheric abundance of stars, i.e. starting with the oxygenrich $(\mathrm{C} / \mathrm{O}<1)$ atmosphere of $\mathrm{M}$ giant stars at the commencement of the AGB evolution, stars can become $\mathrm{S}$ stars, and eventually carbon-rich $(\mathrm{C} / \mathrm{O}>1) \mathrm{C}$ stars (Nowotny et al. 2001). Observational studies of AGB and C stars in Local Group galaxies have been successfully performed in the last few years using a narrowband, i.e. $\mathrm{TiO}$ and $\mathrm{CN}$, photometric technique to separate $\mathrm{C}$ stars from the $\mathrm{M}$ giants among the AGB stars (Albert et al. 2000; Battinelli \& Demers 2000; Nowotny et al. 2001; Demers \& Battinelli 2002; Letarte et al. 2002; Battinelli et al. 2003; Demers et al. 2003a,b; Nowotny et al. 2003; Battinelli \& Demers 2004a,b,c; Harbeck et al. 2004; Kerschbaum et al. 2004). Aside from the narrowband 
photometry, the reddest extreme AGB C stars can be easily distinguished from the $\mathrm{M}$ giants of AGB stars in the broadband near-infrared photometry with a moderately short exposure (Wood et al. 1985; Hughes \& Wood 1990; Frogel et al. 1990; Davidge 2003; Kang et al. 2005). Recent wide field nearinfrared photometric observations of an AGB population in the Magallanic Clouds from 2MASS and DENIS surveys also revealed the presence of a red plume of $\mathrm{C}$ stars in $(K, J-K)$ color-magnitude diagram (Cioni et al. 1999; Nikolaev \& Weinberg 2000).

This is the second in a series of papers studying AGB and $\mathrm{C}$ stars in dwarf galaxies of the Local Group in nearinfrared $J, H$ and $K^{\prime}$ photometry. In the first paper, Kang et al. (2005) presented the near-infrared photometric properties of AGB stars in NGC 185, and demonstrated that it is possible to distinguish $\mathrm{C}$ stars from AGB stars using the near-infrared regime. Kang et al. (2005) also provided near-infrared photometric properties of $73 \mathrm{C}$ stars in NGC 185, and suggested that star formation in the galaxy has a wide range of ages with two different epochs of AGB star formation. In this paper, deep $J, H$ and $K^{\prime}$ images are used to conduct the first investigation of resolved AGB stellar contents in NGC 147 at near-infrared wavelengths. Until now, near-infrared population studies of AGB and $\mathrm{C}$ stars in dwarf galaxies have been performed only for three nearby galaxies, NGC 205 (Davidge 2003), NGC 6822 (Cioni \& Habing 2005) and NGC 185 (Kang et al. 2005).

NGC 147 is a well-known dwarf elliptical satellite of the Andromeda galaxy. NGC 147 has been assumed to be a gravitationally bound pair with NGC 185 (van den Bergh 1998) on the basis of mass estimates and nearly equal mean apparent magnitudes of RR Lyrae variables in both galaxies (Saha et al. 1990; Saha \& Hoessel 1990). Bender et al. (1991) found radial velocities of $-193 \pm 3 \mathrm{~km} \mathrm{~s}^{-1}$ for NGC 147 and $-202 \pm 3 \mathrm{~km} \mathrm{~s}^{-1}$ for NGC 185 with a slight difference of $9 \pm 4 \mathrm{~km} \mathrm{~s}^{-1}$. Nevertheless, the properties of the interstellar medium and stellar contents are different. Whereas NGC 147 is free of gas and dust, its companion NGC 185 contains a significant amount of gas and dust (Young \& Lo 1997). There is no evidence for young stars in NGC 147, but NGC 185 contains a young blue population in the galactic center (Martínez-Delgado et al. 1999). Battinelli \& Demers (2004b,c) suggested that intermediate-age population in NGC 185 is more concentrated than the old population, while those in NGC 147 are spatially well mixed. Metallicities are not identical as well. From deep HS T VI images of NGC 147, Han et al. (1997) determined the metallicity $[\mathrm{Fe} / \mathrm{H}]$ to be -0.91 in the central field and -1.00 in the outer field of the galaxy. Davidge (1994) also found that the central region of NGC 147 contains moderately metalpoor stars with a mean value of $[\mathrm{Fe} / \mathrm{H}] \sim-1$ spanning in the range of $-1.3 \leq[\mathrm{Fe} / \mathrm{H}] \leq-0.7$. However, Martínez-Delgado \& Aparicio (1998) determined a mean metallicity of NGC 185 to be $[\mathrm{Fe} / \mathrm{H}]=-1.43 \pm 0.15$ which decreases to -1.76 for regions further away from the galaxy center. Therefore, the current near-infrared photometry of AGB and C stars in NGC 147, compared to those of NGC 185 (Kang et al. 2005), will provide useful constraints of star formation and stellar evolution in the gravitationally bound galactic system with different environments. On the one hand, Nowotny et al. (2003) and
Table 1. Observational log.

\begin{tabular}{ccccc}
\hline \hline RA $(\mathrm{J} 2000)$ & Dec $(\mathrm{J} 2000)$ & Filter & Exp. time $(\mathrm{s})$ & FWHM $\left(^{\prime \prime}\right)$ \\
\hline $00^{\mathrm{h}} 38^{\mathrm{m}} 52^{\mathrm{s}}$ & $48^{\circ} 20^{\prime} 18^{\prime \prime}$ & $J$ & $8 \times 30$ & 0.65 \\
& & $H$ & $8 \times 30$ & 0.64 \\
& & $K^{\prime}$ & $8 \times 30$ & 0.64 \\
\hline
\end{tabular}

Battinelli \& Demers (2004c) identified 146 and 288 C stars, respectively, in NGC 147, from four color CCD photometry taking advantage of narrowband $\mathrm{TiO}$ and $\mathrm{CN}$.

Section 2 presents $J, H$ and $K^{\prime}$ observations of NGC 147, the data reduction procedure and photometric measurements of AGB stars in NGC 147. The color-magnitude diagrams (CMDs) and color-color diagrams of AGB stars are presented in Sect. 3. In Sect. 4, we discuss the photometric properties of the identified $\mathrm{C}$ stars, the color distributions and luminosity functions of AGB stars, and compare the brightness of AGB tips with theoretical isochrone models in order to discuss the AGB star formation history in NGC 147. A summary of the results is given in Sect. 5 .

\section{Observations, data reduction and photometric measurements}

Observations were obtained with the $3.6 \mathrm{~m}$ Canada-FranceHawaii Telescope at Mouna Kea during the night of UT June 5, 2004. Using the CFHTIR imager mounted on the $\mathrm{f} / 8$ Cassegrain focus of the CFHT, the field centered on NGC 147 was observed with $J, H$ and $K^{\prime}$ filters. The CFHTIR contains a $1024 \times 1024 \mathrm{Hg}: \mathrm{Cd}: \mathrm{Te}$ array with an angular scale of $0.211 /$ pixel, so that each image covers a total field-of-view of $3.6 \times 3{ }^{\prime} 6$ in the sky. The total exposure time was $240 \mathrm{~s}$ per filter for the complete observing sequence of eight 30 second exposures with a four-point square dither pattern. The summary of the run is presented in Table 1.

The data were reduced using the following sequence: (1) the subtraction of dark frames, that were recorded at the beginning and the end of the run, (2) the division by dome flats, that were constructed by subtracting the exposures of dome white spots taken with the lamps off from those recorded with the lamps on, (3) the subtraction of DC sky level, which was estimated by taking the mode of pixel intensity distribution in each flat-fielded frame, and (4) the subtraction of the thermal signature, which was constructed by the median combined flat-fielded images of blank sky regions. The processed target images were aligned to correct the dither offsets, mediancombined, and cropped to the region having full $240 \mathrm{~s}$ of exposure time. The seeings, as measured from the reduced images, are $\sim 00^{\prime} 65 F W H M$ in $J, H$ and $K^{\prime}$ images.

We note that the $K^{\prime}$ filter of CFHTIR has a central wavelength of $2.12 \mu \mathrm{m}$, slightly shorter than those of the standard $K(2.20 \mu \mathrm{m})$ and $K_{\mathrm{S}}(2.15 \mu \mathrm{m})$ filters. This leads to a lower thermal component of the sky background in $K^{\prime}$ and deeper imaging with the same integration time than in the standard $K$ and $K_{\mathrm{S}}$ filters (Wainscoat \& Cowie 1992; Simons \& Tokunaga 2002; Tokunaga et al. 2002). The $K^{\prime}$ filter shows a zero point $0.03 \sim 0.04$ mag fainter than the zero point for the standard $K$ filter (Wainscoat \& Cowie 1992), indicating 
that the difference does not seriously affect the limits of magnitude and colors for the definition of AGB stellar populations in the current study. Hereafter, the $K^{\prime}$ filter is simply referred to as $K$.

The point-spread function fitting routine DAOPHOT II/ ALLSTAR (Stetson 1987; Stetson \& Harris 1988) was used to measure the brightness of individual objects in NGC 147. Instrumental magnitudes were calibrated by the observation of UKIRT faint standard stars (Hawarden et al. 2001). A total of 7303 stars were detected in all three filters and considered as subjects for subsequent analysis.

\section{Photometric properties of AGB stars}

\subsection{Completeness, reddening and adopted distance}

The completeness fractions and uncertainties in photometric measurements were estimated from artificial star experiments. The results from 1500 artificial stars are summarized in Fig. 1. Completeness is defined as the recovery rate of the input artificial stars, $\Delta M$ is the rms difference in magnitudes $(\Delta M)$ between the input and the measured brightnesses, and $\sigma$ is the standard deviation of $\Delta M$. Incompleteness becomes significant when $J, H$ and $K$ are fainter than 19.5, 18.7 and 18.5 mag, respectively, beyond which the recovery rates of input artificial stars are less than $\sim 90 \%$.

The color excess toward NGC 147 is not negligible because of its low Galactic latitude $\left(b=-14^{\circ}\right)$ and patchness of dust and gas. Schlegel et al. (1998) obtained the reddening value $E(B-V)=0.173$ toward NGC 147 from the dust infrared emission feature on the COBE and IRAS maps of $100 \mu \mathrm{m}$. Applying the reddening ratios of Schlegel et al. (1998), the interstellar absorptions in $J H K$ bands are estimated to be $A_{J}=0.156$, $A_{H}=0.100$ and $A_{K}=0.064$. The calculated reddening values are then $E(J-H)=0.056, E(J-K)=0.092$ and $E(H-K)=0.036$.

Mould et al. (1983) calculated the distance modulus of NGC 147 to be $24.0 \pm 0.15$, based on the TRGB method. From the mean magnitude and the luminosity-metallicity relation of type $a b$ RR Lyrae variable stars in NGC 147, Saha et al. (1990) estimated a distance modulus of $23.92 \pm 0.25$. Davidge (1994) used the TRGB method on the VI CCD photometry of NGC 147 to estimate the distance modulus of 24.3. From the re-evaluation of the luminosity-metallicity relations of HB stars and TRGB, Han et al. (1997) estimated the mean distance modulus of NGC 147 to be $24.39 \pm 0.05$ and we adopt this value.

\subsection{Color-magnitude diagrams and color-color diagrams}

Figure 2 shows near-infrared CMDs of stars detected in NGC 147. The upper diagrams are $(K, J-K)$ and $(K, H-K)$ CMDs of stars in the total observed area, and the lower diagrams are those for stars outside the core radius $\left(r \sim 70^{\prime \prime}\right.$, Mateo 1998) of NGC 147. It is apparent that the CMDs contain bright AGB and red giant branch (RGB) stars. The bright part for AGB stars has three components: (1) a bright blue sequence, (2) a vertical sequence with oxygen-rich $\mathrm{M}$ giant stars

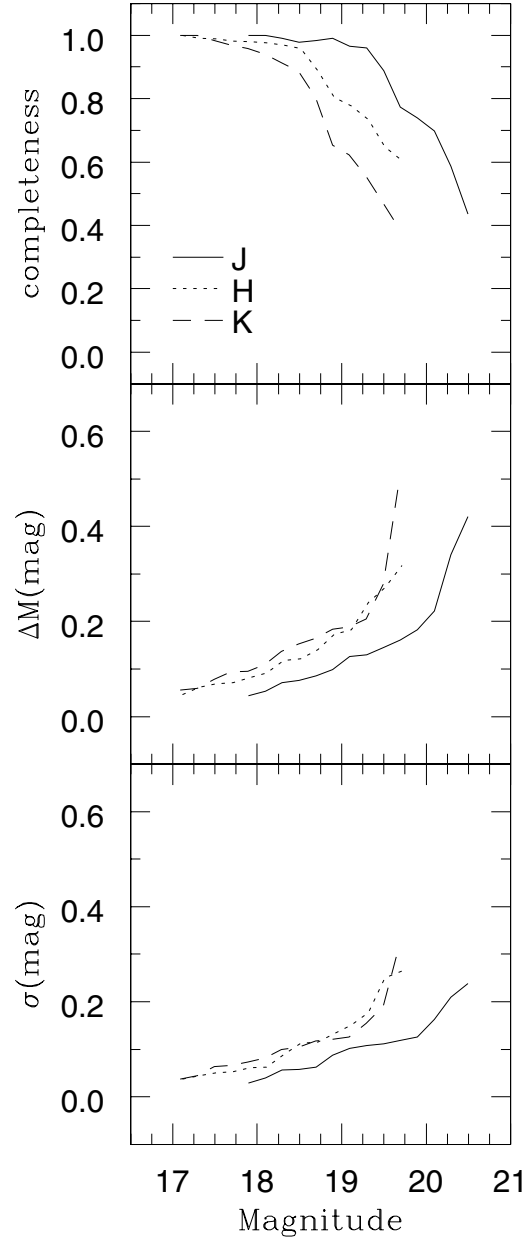

Fig. 1. The results from artificial star experiments. Solid, dotted and dashed lines represent $J, H$ and $K$ bands, respectively. Completeness is defined as the number of recovered artificial stars divided by the total number of stars added. $\Delta M(\mathrm{mag})$ is the mean difference between the actual input magnitudes and the measured magnitudes by DAOPHOT II/ALLSTAR, while $\sigma(\mathrm{mag})$ is the standard deviation of the $\Delta M$.

and (3) a red plume with carbon-rich C stars. No significant difference between shapes of the CMDs for stars in total and in the outer regions indicates that the blending effect of stars is negligible throughout the observed area.

A distinctive group of bright blue stars is located at $0.24 \lesssim$ $(J-K) \lesssim 0.93$ and $(H-K) \sim 0.07$ in Fig. 2. Most of these are considered to be foreground field stars toward NGC 147, similar to those of its companion NGC 185 (Kang et al. 2005). This is consistent with the Galactic star count model of Ratnatunga $\&$ Bahcall (1985), which predicted $~ 30$ bright blue foreground stars with $(B-V)<1.3$ and $V<22$ toward NGC 147 in the field-of-view of the CFHTIR imager. Some of the young blue supergiant stars and evolved bright blue AGB stars in NGC 147 are also possibly among them (Nowotny et al. 2003; Kang et al. 2005).

Using the empirical relations between RGB tip brightness in near-infrared bands and metallicity for stars in a galaxy (Valenti et al. 2004), we estimated the range of magnitude and color of the RGB tip for a given metallicity range $(-1.3 \leq$ $[\mathrm{Fe} / \mathrm{H}] \leq-0.7$, Davidge 1994) of NGC 147. The thick lines 


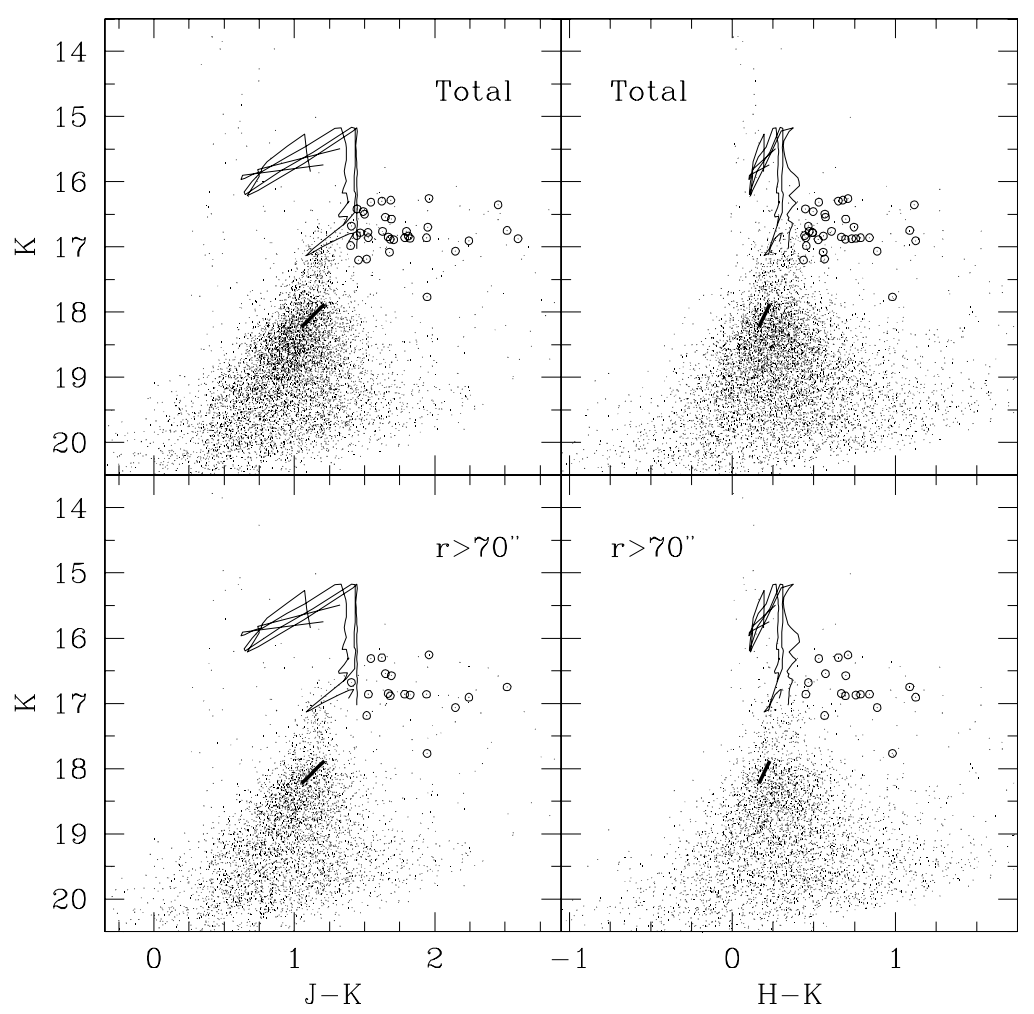

Fig. 2. $(K, J-K)$ and $(K, H-K)$ CMDs of the total observed area (upper) and the outer region (lower) of NGC 147. The loci with thin lines trace the photometric evolution of AGB tip in the range of $\log \left(t_{\mathrm{yr}}\right)=7.8 \sim 10.2$ with $Z=0.004,0.008$ and 0.019 , respectively (Girardi et al. 2002). The color and magnitude range of the RGB tip at the distance of NGC 147 is also presented with a thick line. Open circles indicate $34 \mathrm{C}$ stars cross-identified in both near-infrared photometry (this paper) and $\mathrm{TiO}$ and $\mathrm{CN}$ photometry (Nowotny et al. 2003).

in Fig. 2 are loci of the estimated RGB tip with $17.88<K<$ $18.23,1.05<(J-K)<1.21$ and $0.16<(H-K)<0.23$, at the adopted distance (Sect. 3.1) of NGC 147. In the derived magnitude range of the RGB tip, we appropriately assign AGB stars to be brighter than $K=18 \mathrm{mag}$. The $\mathrm{M}$ giants of AGB stars are considered to be located above the RGB tip vertically extending up to the brightness of the AGB tip. The loci of thin lines in Fig. 2 indicate photometric evolution of the AGB tips in the range of $\log \left(t_{\mathrm{yr}}\right)=7.8 \sim 10.2$ with $Z=0.004,0.008$ and 0.019 (Girardi et al. 2002).

Kang et al. (2005) assigned the color criteria of $(J-K)>$ 1.70 and $(H-K)>0.54$ to $\mathrm{C}$ stars in NGC 185 , adopting color limits for C stars proposed by Wood et al. (1985) and Hughes \& Wood (1990). To assign the color limits of AGB C stars in NGC 147, we first compare the difference of color distributions for AGB stars in NGC 147 (see Fig. 5 in Sect. 4.2) and in NGC 185 (Kang et al. 2005). The comparison shows that the most probable points of color distributions for AGB stars in NGC 147 are bluer than in NGC 185 with color shifts by $\sim 0.35$ in $(J-K)$ and $\sim 0.10$ in $(H-K)$. This trend is also expected from the finding that the apparent integrated colors of NGC 147 in visible bands are bluer than those of NGC 185 (Mateo 1998). Subtracting the estimated color shifts from the color limits of C stars in NGC 185 (Kang et al. 2005), we determine the color criteria for AGB C stars in NGC 147 to be $(J-K)>1.34$ and $(H-K)>0.44$, while there may be some $\mathrm{S}$ type star candidates (Brewer et al. 1996; Letarte et al. 2002).
Color-color diagrams in near-infrared bands are diagnostic tools to identify C star candidates from AGB stars (Frogel et al. 1980; Wood et al. 1985; Marigo et al. 2003). Figure 3 shows the near-infrared color-color diagrams of $(H-K)-(J-H)$ and $(H-K)-(J-K)$ planes for AGB stars brighter than $K=18$ in NGC 147. The C stars with $(J-K)>1.34,(H-K)>0.44$ and $(J-H)>0.91$ are well separated from the bulk of $\mathrm{M}$ giant stars in color-color diagrams. The loci in color-color diagrams follow the color sequence of the AGB tip in Fig. 2, and stars in the blue tails of the color-color diagram are foreground stars that appear in near-infrared CMDs. Finally, total $91 \mathrm{C}$ stars were identified from the near-infrared color-color diagrams for bright AGB stars in NGC 147. The equatorial coordinates and near-infrared photometric properties of the $91 \mathrm{C}$ stars are listed in Table 2.

\section{Discussion}

\section{1. $C$ stars and $C / M$ ratio}

Near-infrared survey of $\mathrm{C}$ stars in nearby galaxies can be obtained with moderately short exposures, and will provide an efficient mean of identifying $\mathrm{C}$ stars from $\mathrm{M}$ giants of AGB stars (Davidge 2003; Marigo et al. 2003; Kang et al. 2005). For the $91 \mathrm{C}$ stars identified in NGC 147, the mean magnitude and colors with standard deviations are estimated to be $\langle K\rangle=$ $16.90 \pm 0.47,\langle(J-K)\rangle=1.91 \pm 0.46$ and $\langle(H-K)\rangle=0.77 \pm 0.31$. These correspond to $\left\langle M_{K}\right\rangle=-7.56,\left\langle(J-K)_{0}\right\rangle=1.81$ and 
Table 2. Coordinates, near-infrared photometric properties of $91 \mathrm{C}$ stars and color indices in VRI bands of cross-identified C stars. The full table is available in electronic form at the CDS.

\begin{tabular}{|c|c|c|c|c|c|c|c|c|c|c|c|c|}
\hline ID & RA(J2000) & $\operatorname{Dec}(\mathrm{J} 2000)$ & $K$ & $\sigma_{K}$ & $J-K$ & $\sigma_{(J-K)}$ & $H-K$ & $\sigma_{(H-K)}$ & $(V-i)_{0}^{a}$ & $\mathrm{ID}_{N}{ }^{a}$ & $R-I^{b}$ & $\mathrm{ID}_{B}^{b}$ \\
\hline 1 & 003306.50 & +483113.3 & 17.250 & 0.105 & 1.825 & 0.322 & 0.859 & 0.153 & & & & \\
\hline 2 & 003306.57 & +483134.2 & 17.766 & 0.036 & 1.945 & 0.123 & 0.983 & 0.097 & 2.01 & 40 & & \\
\hline 3 & 003306.68 & +482839.9 & 17.129 & 0.030 & 2.637 & 0.210 & 1.122 & 0.060 & & & & \\
\hline
\end{tabular}

a Nowotny et al. (2003).

${ }^{b}$ Battinelli \& Demers (2004c).

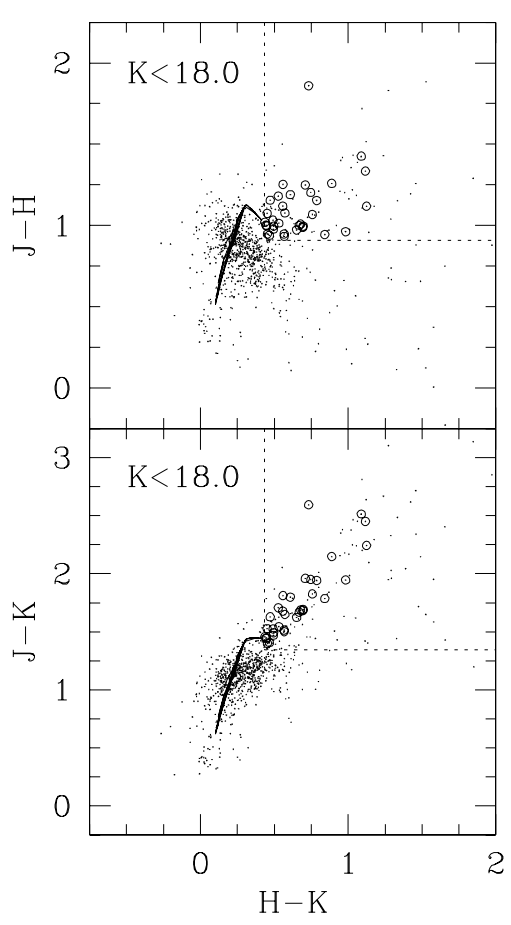

Fig. 3. $(H-K)-(J-H)$ and $(H-K)-(J-K)$ color-color diagrams for AGB stars brighter than $K=18$. Dotted boxes contain C stars with $(J-K)>1.34$ and $(H-K)>0.44$. The loci are the color sequences of the tip of AGB stars as in Fig. 2. Open circles are $34 \mathrm{C}$ stars crossidentified in both near-infrared photometry (this paper) and $\mathrm{TiO}$ and $\mathrm{CN}$ bands photometry (Nowotny et al. 2003).

$\left\langle(H-K)_{0}\right\rangle=0.74$ with the adopted distance modulus and reddening values given in Sect. 3.1.

Battinelli \& Demers (2004c) recently searched for C stars in Local Group galaxies, and they found $288 \mathrm{C}$ stars in the $42^{\prime} \times$ $28^{\prime}$ field centered on the NGC 147 with the two narrowband filters $\mathrm{TiO}$ and CN. Nowotny et al. (2003) also found $146 \mathrm{C}$ stars in the $6.5 \times 6.5$ field in the central region of NGC 147 using a technique similar to Battinelli \& Demers (2004c). We cross-identified $\mathrm{C}$ stars detected in the near-infrared bands with those found by Battinelli \& Demers (2004c) and Nowotny et al. (2003) and found 14 and 34 stars in common. The $34 \mathrm{C}$ stars cross-identified from Nowotny et al. (2003) are shown with open circles in Figs. 2 and 3. Columns 10 and 12 in Table 2 list the optical $(V-i)_{0}$ and $(R-I)$ colors of cross-identified C stars. The ID numbers of stars in Nowotny et al. (2003) and Battinelli \& Demers (2004c) are also listed in Cols. 11 and 13 of Table 1 . Figure 4 shows color-color diagrams of $J H K$ and

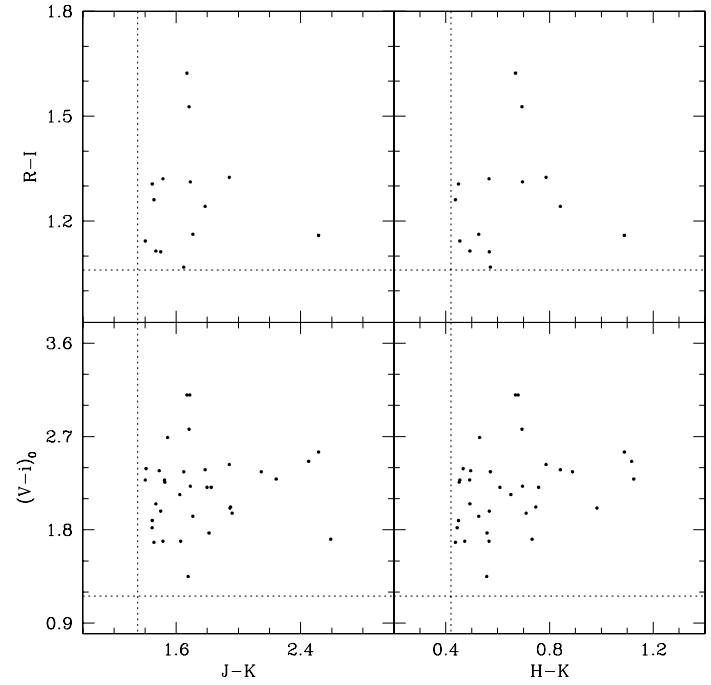

Fig. 4. Color-color diagrams of $J H K$ and $V R I$ bands for 34 crossidentified $C$ stars with $(V-i)_{0}$ indices from Nowotny et al. (2003) and 14 cross-identified $\mathrm{C}$ stars with $(R-I)$ indices from Battinelli \& Demers $(2004 \mathrm{c})$. Vertical dotted lines are the near-infrared color ranges of $\mathrm{C}$ stars, i.e. $(J-K)>1.34$ and $(H-K)>0.44$, while horizontal dotted lines are the color ranges of $\mathrm{C}$ stars in $V R I$ bands, i.e. $(V-i)_{0}>1.16$ and $(R-I)>1.06$, given by Nowotny et al. (2003) and Battinelli \& Demers (2004c).

VRI bands for the cross-identified C stars. The vertical dotted lines are the near-infrared color range of $\mathrm{C}$ stars in this paper, and the horizontal dotted lines are VRI color range of $\mathrm{C}$ stars in Nowotny et al. (2003) and Battinelli \& Demers (2004c). The estimated mean absolute magnitude and colors with standard deviations in the near-infrared band for the $34 \mathrm{C}$ stars crossidentified with Nowotny et al. (2003) are $\left\langle M_{K}\right\rangle=-7.69 \pm 0.31$, $\left\langle(J-K)_{0}\right\rangle=1.66 \pm 0.32$ and $\left\langle(H-K)_{0}\right\rangle=0.62 \pm 0.20$, and for the $14 \mathrm{C}$ stars cross-identified with Battinelli \& Demers $(2004 \mathrm{c})$ are $\left\langle M_{K}\right\rangle=-7.65 \pm 0.24,\left\langle(J-K)_{0}\right\rangle=1.58 \pm 0.29$ and $\left\langle(H-K)_{0}\right\rangle=0.60 \pm 0.18$.

Nowotny et al. (2003) and Battinelli \& Demers (2004b,c) found the mean absolute magnitudes $\left\langle M_{I}\right\rangle$ of $\mathrm{C}$ stars in NGC 147 and NGC 185 to be identical. The obtained mean near-infrared absolute magnitude and colors with standard deviations for C stars in NGC 147 seem to be comparable to the mean values of $73 \mathrm{C}$ stars in NGC 185 (Kang et al. 2005), i.e. $\left\langle M_{K}\right\rangle=-7.93 \pm 0.38,\left\langle(J-K)_{0}\right\rangle=2.25 \pm 0.46$ and $\left\langle(H-K)_{0}\right\rangle=0.87 \pm 0.25$. It is worth noting here that the brighter $\left\langle M_{K}\right\rangle$ of C stars in NGC 185 compared to the NGC $147 \mathrm{C}$ stars, without considering the standard deviation of the $\left\langle M_{K}\right\rangle \mathrm{s}$, could 
Table 3. The number of $\mathrm{C}$ stars and $\mathrm{M}$ giants and $\mathrm{C} / \mathrm{M}$ ratios of NGC 147.

\begin{tabular}{ccc}
\hline \hline & NGC 147 & NGC 185 \\
\hline $\mathrm{C}$ & 91 & 73 \\
$\mathrm{M}$ & 555 & 645 \\
$\mathrm{C} / \mathrm{M}$ & $0.16 \pm 0.02$ & $0.11 \pm 0.01$ \\
\hline $\mathrm{C} / \mathrm{M}^{a}$ & 0.154 & 0.089 \\
$\mathrm{C} / \mathrm{M}^{b}$ & $0.24 \pm 0.02$ & $0.17 \pm 0.02$ \\
\hline${ }^{a}$ Nowotny et al. (2003). \\
${ }^{b}$ Battinelli \& Demers (2004c).
\end{tabular}

be due to a possible population of younger $\mathrm{C}$ stars in the central region of NGC 185 where some recent star formation has taken place (Martínez-Delgado \& Aparicio 1999). Indeed, one would expect $\mathrm{C}$ stars with different ages in the ongoing star formation area in a galaxy (Letarte et al. 2002; Battinelli \& Demers 2004b), and the luminosity and color of C stars depend on their ages (Da Costa 1994; Mouhcine \& Lançon 2003). The different intrinsic properties of galaxies, such as the spatial distribution of interstellar medium and metallicity, could also affect the photometric properties of the $\mathrm{C}$ star population.

We evaluated the number ratio of $\mathrm{C}$ stars to $\mathrm{M}$ giants $(\mathrm{C} / \mathrm{M})$ of the AGB population in NGC 147. To minimize the effect of the bright blue foreground stars on the total counts of $\mathrm{M}$ giant stars, we counted $\mathrm{M}$ giant stars with $15.5<K<18.0$ and $0.93<(J-K)<1.34$ on the $(K, J-K)$ CMD. The lower and upper limits of $K$ and $(J-K)$ for $\mathrm{M}$ giant stars correspond to the magnitudes of AGB and RGB tips and the color limits of foreground stars and $\mathrm{C}$ stars, respectively. Possible RGB stars with $M_{\text {bol }}$ (see Sect 4.3 for the bolometric correction) fainter than the bolometric luminosity of the RGB tip were selected and removed from the counted $\mathrm{M}$ giant stars in $(K, J-K)$ CMD (cf. Battinelli et al. 2003). Then the estimated C/M ratio for the total observed area from the near-infrared photometry of NGC 147 is $0.16 \pm 0.02$, which is comparable to those of 0.154 from Nowotny et al. (2003) and 0.24 \pm 0.02 from Battineli $\&$ Demers (2004c). The local C/M ratios are estimated to be $0.14 \pm 0.02$ for the inner region $\left(r<70^{\prime \prime}\right)$ and $0.19 \pm 0.03$ for the outer region $\left(r>70^{\prime \prime}\right)$, indicating a slight radial gradient. Battinelli \& Demers (2004c) also found that the local values of $\mathrm{C} / \mathrm{M}$ increase from the center up to the 3.5 scale length $\left(\sim 12^{\prime}\right)$ of NGC 147 , in agreement with the metallicity gradient found by Han et al. (1997). Contrary to NGC 147, Kang et al. (2005) found no apparent $\mathrm{C} / \mathrm{M}$ radial gradient at the central region of NGC 185 from the near-infrared photometry (see also Battinelli \& Demers 2004b).

Table 3 summarizes the number of $\mathrm{C}$ stars and $\mathrm{M}$ giants, and C/M ratios in NGC 147 and NGC 185 derived from nearinfrared photometry. Previously determined $\mathrm{C} / \mathrm{M}$ ratio of the galaxies are also presented in Table 3. Apparently, NGC 147 has $\mathrm{C} / \mathrm{M}$ values larger than NGC 185 for all independent observations, which indicates that the conditions of formation and evolution of $\mathrm{C}$ stars may be different in each galaxy. Indeed, the evolutionary property of $\mathrm{C}$ stars in a galaxy is known to be a function of star formation and metallicity as well (Groenewegen 1999; Battinelli \& Demers 2004b). A more homogeneous data set of C/Ms for the Local Group galaxies estimated from the near-infrared survey would then be a complementary confirmation of such correlations, and the nearinfrared observations of $\mathrm{C}$ stars in many other dwarf galaxies will provide further essential clues to the formation and evolution of AGB C stars.

\subsection{Color distributions}

The color distribution of AGB stars can be used to infer the star formation epoch in a galaxy. Davidge (2003) analyzed the $(J-K)$ histogram distributions of AGB stars in NGC 205 and found that the brightest AGB stars formed within the past $0.1 \mathrm{Gyr}$ and the previous episode of star formation occurred a few tenths of a Gyr earlier. From the $(J-K)$ and $(H-K)$ color distributions of AGB stars in NGC 185 with the predicted colors for $Z=0.019$ isochrones for AGB stars (Girardi et al. 2002), Kang et al. (2005) suggested two epochs of star formation in NGC 185 with a wide range of ages.

Figure 5 shows the histogram distributions of the $(J-K)$ and $(H-K)$ colors in 0.05 mag bins for the AGB stars with $15.5<K<18.0$ in NGC 147 . Both $(J-K)$ and $(H-K)$ histograms have a main peak containing $\mathrm{M}$ giant stars, a red tail containing $C$ stars with a weak excess and a blue excess possibly due to bright blue foreground stars and AGB stars younger than $\mathrm{M}$ giant stars. The color distributions of $\mathrm{M}$ giant stars are represented by eye-fitted Gaussians with $\sigma$ values equal to the random photometric errors, $\sigma_{(J-K)}=0.100$ and $\sigma_{(H-K)}=0.106$, predicted from the artificial star experiments (Fig. 1). The shaded sections in red tails of histograms represent the distribution of $91 \mathrm{C}$ stars with $(J-K)>1.34$, $(H-K)>0.44$ and $(J-H)>0.91$ as defined in Sect. 3.2. A weak excess of AGB stars appears in $(J-K) \gtrsim 1.35$ and $(H-K) \gtrsim 0.40$ on the red tails of the color distributions. The blue excess appears in the color histograms when $(J-K) \lesssim 0.95$ and $(H-K) \lesssim 0.10$.

Arrows in Fig. 5 are the theoretically predicted colors of the AGB tip for $Z=0.019$ isochrones of $\log \left(t_{\mathrm{yr}}\right)=8.1,8.4$ and 9.5 (Girardi et al. 2002) with the adopted distance and reddening of NGC 147. It is apparent that the colors of the M giant peak in the histogram correspond to $\log \left(t_{\mathrm{yr}}\right) \sim 8.4$, and a spread of color distributions of $\mathrm{M}$ giant stars indicates a range of star formation epochs in NGC 147, i.e. $\sim 8.2 \lesssim \log \left(t_{\mathrm{yr}}\right) \lesssim 8.6$. Kang et al. (2005) found that there were two possible epochs of star formation for the $\mathrm{M}$ giant stars in NGC 185, having $\log \left(t_{\mathrm{yr}}\right) \approx 9.0 \sim 9.4$ and $7.8 \sim 8.5$. Then, it is inferred that the ages for most M giants in NGC 147 are similar to the younger $\mathrm{M}$ giants in NGC 185, while their formation occurred more recently than the older M giants in NGC 185 by 2 Gyr. A few older M giants with $\log \left(t_{\mathrm{yr}}\right) \sim 9.5$ in NGC 147 are expected at $(J-K) \sim 1.50$ and $(H-K) \sim 0.50$ on the weak excess of the red tails in the color distributions. If stars in the blue excess in Fig. 5 are not foreground stars but young stars in NGC 147, their ages $\operatorname{are} \log \left(t_{\mathrm{yr}}\right) \lesssim 8.2$, similar to the ages of young blue stars in NGC $185\left(\log \left(t_{\mathrm{yr}}\right) \lesssim 8.5\right.$, Kang et al. 2005). Therefore, the color distributions of AGB stars in NGC 147 indicate that AGB star formation in the galaxy took place over an extend 


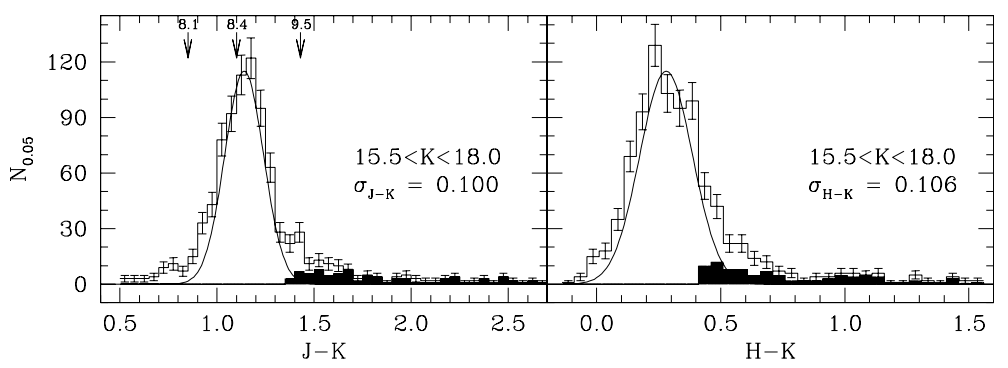

Fig. 5. Histogram distributions of the $(J-K)$ and $(H-K)$ colors of stars with $K$ between 15.5 and 18.0 mag. Arrows mark the theoretically predicted colors of the AGB tip for $Z=0.019$ isochrones of $\log \left(t_{\mathrm{yr}}\right)=8.1,8.4$ and 9.5 (Girardi et al. 2002) at the distance of NGC 147. The shaded sections represent the distribution of the $91 \mathrm{C}$ stars identified.

period of time, similar to the case of NGC 185, while the majority of M giant stars in NGC 147 were formed at the similar star formation epoch of younger M giant stars in NGC 185.

\subsection{Luminosity functions}

Luminosity functions (LFs) for the resolved AGB stars in a galaxy is one of the most important concept used for comparing models of $\mathrm{M}$ giant and $\mathrm{C}$ star evolution with observations. Indeed, it seems that bolometric LF and the mean bolometric magnitude of $\mathrm{C}$ stars in a galaxy depend on metallicity and the star formation history of a galaxy (e.g. Nowotny et al. 2003; Groenewegen 1999). Battinelli \& Demers (2004c) suggested that the $I$ band LF of AGB stars can be used as a standard candle to estimate the distance of galaxies. Near-infrared observations have the advantage of deriving the LFs of AGB stars in a galaxy rather than the visible band observations, as Freedman (1992) noted that some very red bright stars that are visible on near-infrared images are not always detected on optical band images.

Battinelli \& Demers (2004c) showed that the $I$ band LF of C stars in NGC 147 is well represented by a relatively narrow Gaussian, from which they found that the mean apparent magnitude of the C stars in NGC 147 is $\langle I\rangle=20.31 \pm 0.40$, corresponding to the mean absolute magnitude of $\left\langle M_{I}\right\rangle=-4.39$. Nowotny et al. (2003) also derived the $i$ band LF of C stars in NGC 147 and estimated the mean $\left\langle M_{i}\right\rangle$ to be $-4.24 \pm 0.42$. Nowotny et al. (2003) derived the bolometric LF for C stars in NGC 147, and found that the shape of the bolometric LF and the mean bolometric magnitude of $\left\langle M_{\mathrm{bol}}\right\rangle=-4.33 \pm 0.47$ are in good agreement with those of the SMC.

Here, we derived the LFs in $M_{K}$ and bolometric LFs for $M$ giants and C stars in NGC 147 from the near-infrared observations to compare with those from the previous visible band photometry. The completeness-corrected LFs in $M_{K}$ for M giant stars and C stars in NGC 147 are shown in the two diagrams on the left in Fig. 6. A two-side Kolmogorov-Smirnov test for the $M_{K}$ LF of C stars and the Gaussian with the mean absolute magnitude and standard deviation of $\left\langle M_{K}\right\rangle=-7.56 \pm 0.47$ (Sect. 4.1) estimated only a $22 \%$ confidence level of having the same distribution between the two. This indicates that the $M_{K}$ LF of C stars in NGC 147 is not likely to be a Gaussian distribution. From the logarithmic LF of M giant stars of NGC 147 in the inner small panel at the lower-left panel of Fig. 6, we estimated the logarithmic slope of the $M_{K} \mathrm{LF}$ for the bright $\mathrm{M}$ giant stars $\left(-8.5<M_{K}<-7.1\right)$ to be $0.79 \pm 0.02$. This value is comparable with the slope for NGC 185 estimated by Kang et al. (2005), i.e. $0.83 \pm 0.02$. As proposed by Kang et al. (2005), the universality of the slope in the $K$ band LF of bright stars in nearby dwarf galaxies can be checked with more near-infrared photometry of the resolved AGB stars in the galaxies.

Bolometric corrections in $K$ band, $B C_{K}$, were estimated separately for each M giant and AGB C star in NGC 147 from the empirical relations between $B C_{K}$ and $(J-K)$ for Galactic and the LMC oxygen-rich M giant AGB stars (Bessell \& Wood 1984) and for C stars in the LMC (Costa \& Frogel 1996). We note relatively large differences and uncertainties for the $B C s$ for $\mathrm{C}$ stars depending on authors (e.g. Bessell \& Wood 1984; Montegriffo et al. 1998). Using the bolometric corrections, the completeness-corrected bolometric LFs for the $\mathrm{M}$ giant stars and the $\mathrm{C}$ stars in NGC 147 were constructed, and represented in the two diagrams on the right in Fig. 6. For the lower limit we followed the usual $M_{\mathrm{bol}}<-3.5$ threshold corresponding to the bolometric magnitude of the tip of RGB (Ferraro et al. 2000). The bolometric LF of M giant stars in NGC 147 extends up to $M_{\text {bol }}=-5.8$ mag as shown in the lower-right panel in Fig. 6, and that of only $\mathrm{C}$ stars in the upper-right panel in Fig. 6 spans $-5.6<M_{\text {bol }}<-3.5$. The estimated mean bolometric magnitude of C stars in NGC 147, i.e. $\left\langle M_{\text {bol }}\right\rangle=-4.32 \pm 0.49$, is in good agreement with the one derived from $V i$ photometry as $-4.33 \pm 0.47$ (Nowotny et al. 2003). This value is also comparable to $\left\langle M_{\mathrm{bol}}\right\rangle=-4.50 \pm 0.42$ for $\mathrm{C}$ stars in its companion NGC 185 (Kang et al. 2005).

\subsection{AGB tip and age}

The brightness of the AGB tip can be used to determine the age of stars in the AGB tip in a galaxy when it is compared to the theoretical isochrones of AGB stars (e.g. Girardi et al. 2002). By applying the predicted relationship between AGB tip brightness and age, Davidge (2003) and Kang et al. (2005) estimated the age of the AGB stars and inferred the star formation epoch in NGC 205 and NGC 185. However, identifying the peak $K$ brightness of AGB stars in a galaxy is not easy due to the difficulties in obtaining large observational samples and the field star contamination. The Galactic star count model (Ratnatunga \& Bahcall 1985) predicts only a few field stars with similar colors and magnitudes of the AGB stars in NGC 147, from 


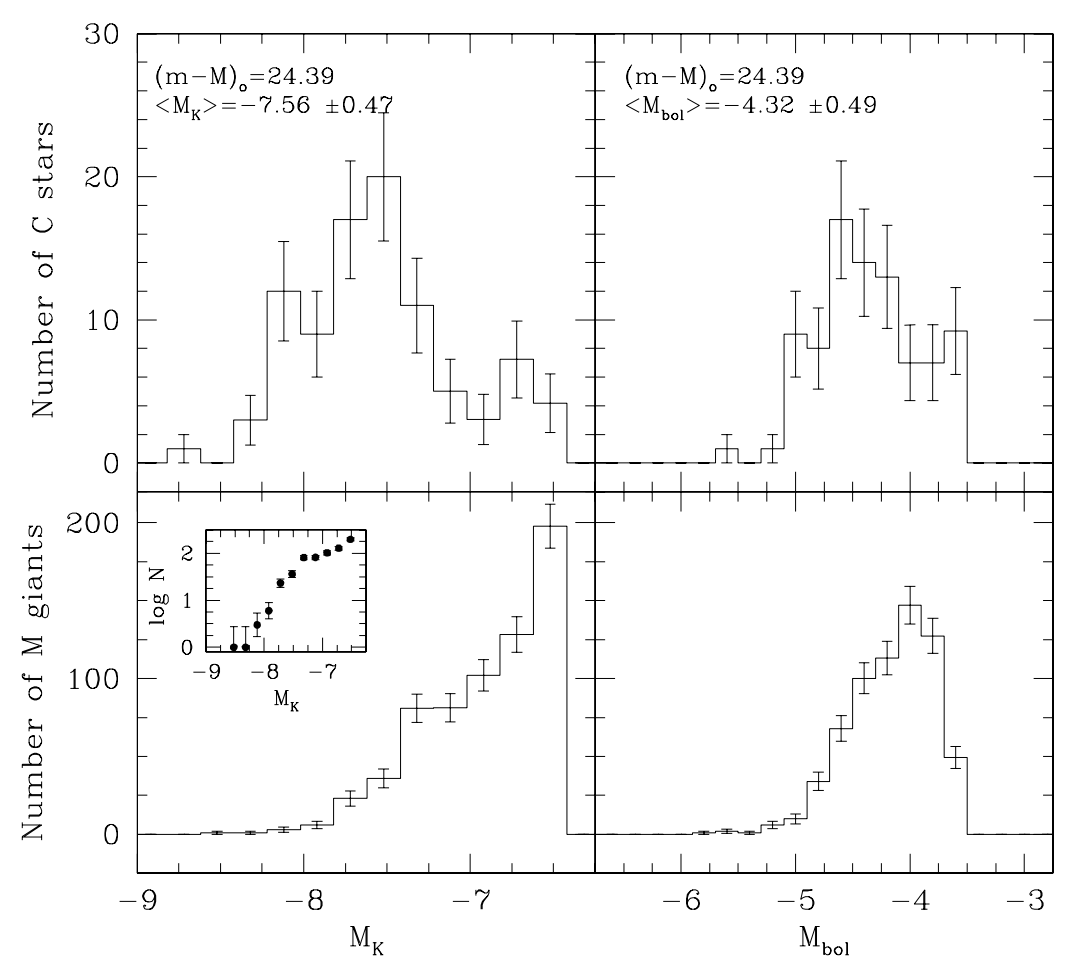

Fig. 6. Left: the completeness corrected LFs in $M_{K}$ for M giant stars and C stars. The inner small panel in the lower panel shows the logarithmic LF of M giant stars in NGC 147. Right: the completeness corrected bolometric LFs for M giant stars and C stars.

which we disregard the field star contamination to determine the AGB tip. The similarity of the bright-end envelope morphologies of CMDs through the observed area (e.g. Fig. 2) and the scattered spatial distribution of bright AGB stars also suggest that the blending effects in our near-infrared images are negligible on the counts for bright AGB stars in NGC 147.

Figure 7 shows the relationship between $K$ brightness of the AGB tip and age at the distance of NGC 147 extracted from isochrones of Girardi et al. (2002) with $Z=0.004,0.008$ and 0.019 . The models predict that the peak AGB brightness in a population with $\log \left(t_{\mathrm{yr}}\right)=10.0$ should occur near $K=16.5 \sim$ 17.0, as indicated by a shadow bar in Fig. 7. Indeed, the excess of a few bright stars in the red tail of the color distribution of AGB stars with $(J-K) \gtrsim 1.35$ and $(H-K) \gtrsim 0.40$ in Fig. 5 corresponds to the AGB tip stars having $K=16.5 \sim 17.0$ in the CMDs of Fig. 2. Also, the models in Fig. 7 predict that stars with $\log \left(t_{\mathrm{yr}}\right) \lesssim 8.2$ would have a peak AGB brightness at $K=15.4 \sim 16.1$. This agrees with the age estimate of stars at the blue excess in the color distribution of Fig. 5 and the peak brightness of them in the CMDs of Fig. 2. On the other hand, the color distributions in Fig. 5 are dominated by $\mathrm{M}$ giant stars that formed during intermediate epochs, i.e. $\log \left(t_{\mathrm{yr}}\right) \sim 8.4$, for which the models predict that the AGB tip brightness would occur at $K=15.5 \sim 16.2$. To confirm this for the stars, we counted the brightest $\mathrm{M}$ giant stars $(K<16.5)$ with $0.93<(J-K)<1.34$ in $(K, J-K)$ CMD of Fig. 2. Only 9 stars were in the range, and the mean $K$ magnitude of the stars was estimated to be $\langle K\rangle=16.1 \pm 0.3$, which is in agreement with the model-predicted value in Fig. 7. Therefore, the model prediction of AGB tip brightness and age in Fig. 7 is consistent with the AGB star formation history in NGC 147 inferred from the color distribution of AGB stars in Fig. 5. It is conclusive that the majority of bright $\mathrm{M}$ giant stars in NGC 147 were formed at a peak $\log \left(t_{\mathrm{yr}}\right) \sim 8.4$ with a wide range of ages. Ages of most M giant stars in NGC 147 are similar to those of the younger $\mathrm{M}$ giants in its companion NGC 185, and their formation occurred more recently than the older $\mathrm{M}$ giants in NGC 185 by 2 Gyr (cf. Kang et al. 2005), as also indicated in the color distribution of the stars.

\section{Summary}

Near-infrared $J, H$ and $K^{\prime}$ images were used to investigate the bright stellar content of the AGB population in the nearby dwarf elliptical galaxy NGC 147 . The results are summarized as follows.

1. The near-infrared CMDs obtained contain RGB and AGB star populations, where the latter consists of a group of bright blue stars, a dominant $\mathrm{M}$ giant population and a red $\mathrm{C}$ star population.

2. A total of $91 \mathrm{C}$ stars are identified from $\mathrm{M}$ giant stars in the near-infrared CMDs and color-color diagrams. The mean magnitude with the standard deviation of the $\mathrm{C}$ stars is $\langle K\rangle=16.90 \pm 0.47$, corresponding to $\left\langle M_{K}\right\rangle=-7.56$, and the mean colors are $\left\langle(J-K)_{0}\right\rangle=1.81,\left\langle(H-K)_{0}\right\rangle=0.74$.

3. The $\mathrm{C} / \mathrm{M}$ ratio of $\mathrm{AGB}$ stars in the total observed area is estimated to be $0.16 \pm 0.02$. The local $\mathrm{C} / \mathrm{M}$ ratios are estimated to be $0.14 \pm 0.02$ for the inner region $\left(r<70^{\prime \prime}\right)$ and $0.19 \pm 0.03$ for the outer region $\left(r>70^{\prime \prime}\right)$, indicating a weak radial gradient.

4. The $(J-K)$ and $(H-K)$ color histograms show a main peak containing $\mathrm{M}$ giant stars, a red tail containing $\mathrm{C}$ stars with 


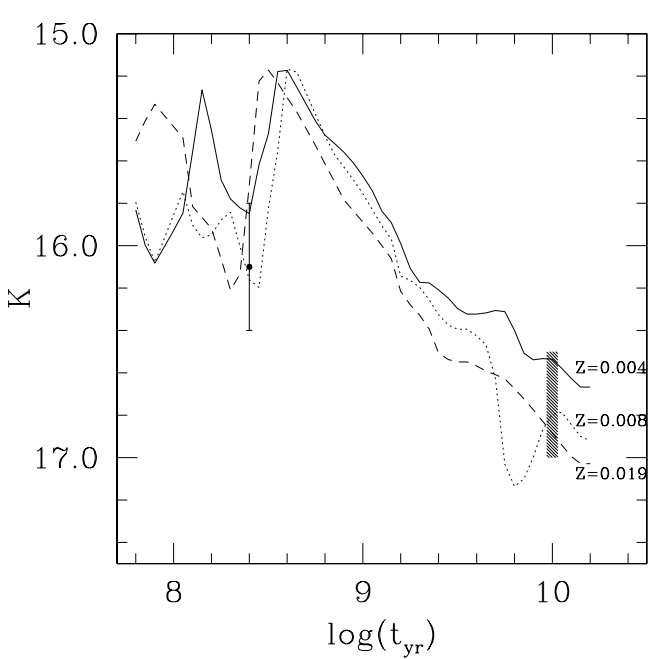

Fig. 7. Relationship between the $K$ magnitude of the AGB tip and age at the distance of NGC 147, as predicted by isochrones (Girardi et al. 2002) for $Z=0.004$ (solid line), 0.008 (dotted line) and 0.019 (dashed line). The shadow bar represents the range of AGB tip brightness for stars having $\log \left(t_{\mathrm{yr}}\right)=10.0$. The estimated mean magnitude of the AGB tip in NGC 147 is also represented by a dot with a measurement error.

a weak excess and a blue excess possibly due to AGB stars younger than $\mathrm{M}$ giants and bright blue foreground stars. The colors of the $\mathrm{M}$ giant peak in the histograms correspond to the age of $\log \left(t_{\mathrm{yr}}\right) \sim 8.4$, and a spread of color distribution of the most $\mathrm{M}$ giant stars indicates a range of star formation epochs in NGC 147 of $\sim 8.2 \lesssim \log \left(t_{\mathrm{yr}}\right) \lesssim 8.6$.

5. The slope of the logarithmic $K$ band LF of M giant stars with $-8.5<M_{K}<-7.1$ is estimated to be $0.79 \pm 0.02$.

6. The bolometric LF of M giant stars in NGC 147 extends up to $M_{\text {bol }}=-5.8$ magnitude, and that of only $\mathrm{C}$ stars spans $-5.6<M_{\mathrm{bol}}<-3.5$. The mean bolometric magnitude of $\mathrm{C}$ stars in NGC 147 is estimated to be $\left\langle M_{\mathrm{bol}}\right\rangle=-4.32 \pm$ 0.49 .

7. Comparison of the theoretical isochrone models with the peak AGB brightness and the color distributions of AGB stars indicates that most of bright $\mathrm{M}$ giants in NGC 147 were formed at $\log \left(t_{\mathrm{yr}}\right) \sim 8.4$ with a range of $8.2 \sim 8.6$. The ages of the majority of M giant stars in NGC 147 are similar to those of younger M giant stars in NGC 185, while their formation occurred more recently than the older M giant stars in NGC 185 by 2 Gyr.

Acknowledgements. We thank the anonymous referee for useful comments that improved this paper. Support for this work was provided by Korea Astronomy and Space Science Institute, for which we are grateful.

\section{References}

Albert, L., Demers, S., \& Kunkel, W. E. 2000, AJ, 119, 2780 Battinelli, P., \& Demers, S. 2000, AJ, 120, 1801 Battinelli, P., \& Demers, S. 2004a, A\&A, 416, 111 Battinelli, P., \& Demers, S. 2004b, A\&A, 417, 479 Battinelli, P., \& Demers, S. 2004c, A\&A, 418, 33 Battinelli, P., Demers, S., \& Letarte, B. 2003, AJ, 125, 1298
Bender, R., Paquet, A., \& Nieto, J.-L. 1991, A\&A, 246, 349

Bessell, M. S., \& Wood, P. R. 1984, PASP, 96, 247

Brewer, J. P., Richer, H. B., \& Crabtree, D. R. 1996, AJ, 112, 491

Cioni, M.-R. L., \& Habing, H. J. 2005, A\&A, 429, 837

Cioni, M.-R. L., Habing, H. J., Loup, C., et al. 1999, in New Views of the Magellanic Clouds, ed. Y.-H. Chu, N. Suntzeff, J. Hesser, \& D. Bohlender, IAU Symp., 190, 385

Costa, E., \& Frogel, J. A. 1996, AJ, 112, 2607

Da Costa, G. S. 1994, in Dwarf Galaxies, Proc. ESO/OHP Workshop, ed. G. M. Meylan, \& P. Prugnel (Garching ESO), ESO Conf. Workshop Proc., 49, 221

Davidge, T. J. 1994, AJ, 108, 2123

Davidge, T. J. 2003, ApJ, 597, 289

Demers, S., \& Battinelli, P. 2002, AJ, 123, 238

Demers, S., Battinelli, P., \& Letarte, B. 2003a, AJ, 125, 3037

Demers, S., Battinelli, P., \& Letarte, B. 2003b, A\&A, 410, 795

Ferraro, F. R., Montegriffo, P., Origlia, L., \& Fusi Pecci, F. 2000, AJ, 119,1282

Freedman, W. D. 1992, AJ, 104, 1349

Frogel, J. A., Persson, S. E., \& Cohen, J. G. 1980, ApJ, 239, 495

Frogel, J. A., Mould, J., \& Blanco, V. M. 1990, ApJ, 352, 96

Girardi, L., Bertelli, G., Bressan, A., et al. 2002, A\&A, 391, 195

Grebel, E. K. 1999, in The Stellar Content of Local Group Galaxies, ed. P. Whitelock, \& R. Cannon, IAU Symp., 192, 17

Groenewegen, M. A. T. 1999, in The Asymptotic Giant Branch Stars, ed. T. Le Berte, A. Lèbre, \& C. Waelkens, IAU Symp., 191, 535

Han, M., Hoessel, J. G., Gallagher, J. S. III, Holtzman, J., \& Stestson, P. B. 1997, AJ, 113, 1001

Harbeck, D., Gallagher, J. S. III, \& Grebel, E. K. 2004, AJ, 127, 2711

Hawarden, T. G., Leggett, S. K., Letawsky, M. B., Ballantyne, D. R., \& Casali, M. M. 2001, MNRAS, 325, 563

Hughes, S. M. G., \& Wood, P. R. 1990, AJ, 99, 784

Iben, I., Jr., \& Renzini, A. 1983, ARA\&A, 21, 271

Kang, A., Sohn, Y.-J., Rhee, J., et al. 2005, A\&A, 437, 61

Kerschbaum, F., Nowotny, W., Olofsson, H., \& Schwarz, H. E. 2004, A\&A, 427, 613

Letarte, B., Demers, S., Battinelli, P., \& Kunkel, W. E. 2002, AJ, 123, 832

Marigo, P., Girardi, L., \& Chiosi, C. 2003, A\&A, 403, 225

Martínez-Delgado, D., \& Aparicio, A. 1998, AJ, 115, 1462

Martínez-Delgado, D., Aparicio, A., \& Gallart, C. 1999, AJ, 118, 2229

Mateo, M. 1998, ARA\&A, 36, 435

Mouhcine, M., \& Lançon, A. 2003, MNRAS, 338, 572

Montegriffo, P., Ferraro, F. R., Origlia, L., \& Fusi Pecci, F. 1998, MNRAS, 297, 872

Mould, J. R., Kristian, J., \& Da Costa, G. S. 1983, ApJ, 270, 471

Nikolaev, S., \& Weinberg, M. D. 2000, ApJ, 542, 804

Nowotny, W., Kerschbaum, F., Olofsson, H., \& Schwarz, H. E. 2003, A\&A, 403, 93

Nowotny, W., Kerschbaum, F., Schwarz, H. E., \& Olofsson, H. 2001, A\&A, 367, 557

Ratnatunga, K. U., \& Bahcall, J. N. 1985, ApJS, 59, 63

Saha, A., \& Hoessel, J. G. 1990, AJ, 99, 97

Saha, A., Hoessel, J. G., \& Mossman, A. E. 1990, AJ, 100, 108

Schlegel, D. J., Finkbeiner, D. P., \& Davis, M. 1998, ApJ, 500, 525

Simons, D. A., \& Tokunaga, A. 2002, PASP, 114, 169

Stetson, P. B. 1987, PASP, 99, 191

Stetson, P. B., \& Harris, W. E. 1988, AJ, 96, 909

Tokunaga, A. T., Simons, D. A., \& Vacca, W. D. 2002, PASP, 114, 180

Tonry, J. L., Dressler, A., Blakeslee, J. P., et al. 2001, ApJ, 546, 681

Valenti, E., Ferraro, F. R., \& Origlia, L. 2004, MNRAS, 354, 815

van den Bergh, S. 1998, AJ, 116,1688

Wainscoat, R. J., \& Cowie, L. L. 1992, AJ, 103, 332

Wood, P. R., Bessell, M. S., \& Paltoglou, G. 1985, ApJ, 290, 477

Young, L. M., \& Lo, K. Y. 1997, ApJ, 476, 127 